QMW-PH-00-13

RU-NHETC-2000-50

hep-th/0011190

\title{
Fivebranes Wrapped On Associative Three-Cycles
}

\author{
Bobby S. Acharya ${ }^{\dagger, 1}$, Jerome P. Gauntlett*,2 and Nakwoo Kim*,3 \\ $\dagger$ Department of Physics \\ Rutgers University \\ 126 Freylinghuysen $R d$ \\ NJ 08854-0849, USA \\ * Department of Physics \\ Queen Mary, University of London \\ Mile End Rd, London E1 4NS, UK
}

\begin{abstract}
We construct supergravity solutions corresponding to fivebranes wrapping associative three-cycles of constant curvature in manifolds of $G_{2^{-}}$ holonomy. The solutions preserve 2 supercharges and are first constructed in $D=7$ gauged supergravity and then lifted to $D=10,11$. We show that the low-energy theory of M-fivebranes wrapped on a compact hyperbolic three-space is dual to a superconformal field theory in $\mathrm{D}=3$ by exhibiting a flow to an $A d S_{4}$ region. For IIB-fivebranes wrapped on a three-sphere we speculate on a connection with spontaneous supersymmetry breaking of pure $\mathcal{N}=1$ super Yang-Mills theory in $\mathrm{D}=3$.
\end{abstract}

1 E-mail: bacharya@physics.rutgers.edu

2 E-mail: j.p.gauntlett@qmw.ac.uk

${ }^{3}$ E-mail: n.kim@qmw.ac.uk 


\section{Introduction}

When a brane wraps a supersymmetric cycle one typically finds a "twisted" field theory realised on the worldvolume of the brane [1]. One way to see this is to note that the cycle will typically not have a covariantly constant spinor and hence supersymmetry must be realised in some twisted fashion. The transverse fluctuations of the brane are specified by sections of the normal bundle of the brane worldvolume and it is the structure of this bundle that gives rise to the twisting.

An investigation of the supergravity/string-theory duals of such theories was presented in [2, 3] (for related work see [⿷, 5, 6, 7, 8, 9, 10, 11]). Consider a supersymmetric spacetime of the form $\mathbb{R}^{1, q} \times M$ and a $q+p$-brane wrapping a supersymmetric $p$-cycle $\Sigma_{p} \subset M$. After taking an appropriate limit to decouple gravity while keeping the volume of $\Sigma_{p}$ fixed [2, 12, 13], one obtains a twisted field theory on the worldvolume of the brane $\mathbb{R}^{1, q} \times \Sigma_{p}$. It was argued in [2] that in this decoupling limit the field theory is insensitive to the global geometry of $M$ : its effect is local and simply determines the specific twisted field theory. At energies low compared to the inverse size of $\Sigma_{p}$, these theories then reduce to $D=(q+1)$-dimensional field theories. If we have a large number of branes wrapping the cycle we might expect to be able to find supergravity duals for these theories.

The cases analysed in [2] correspond to M-fivebranes and D3-branes wrapping Riemann-surfaces that are holomorphically embedded in Calabi-Yau two- or threefolds. These give rise to four-dimensional field theories with $\mathcal{N}=2,1$ supersymmetry and two dimensional field theories with $(4,4),(2,2)$ supersymmetry, respectively. One interesting feature of this work is that supergravity solutions were found with $A d S_{5}$ and $A d S_{3}$ regions in the IR, respectively, providing new AdS/CFT examples. In subsequent work IIB fivebranes wrapped on two-spheres leading to $\mathcal{N}=1$ supersymmetry in four dimensions were studied [3]. A fascinating aspect of this work is that it seems to provide a starting point for analysing the large $\mathrm{N}$-limit of pure $\mathcal{N}=1$ supersymmetric Yang-Mills (SYM) theory in four-dimensions.

It is natural to extend these investigations by trying to construct supergravity duals corresponding to branes wrapping higher dimensional supersymmetric cycles. The examples we will focus on in this paper are M-fivebranes or IIB fivebranes wrapping associative 3-cycles in seven-dimensional manifolds with $G_{2}$-holonomy. These configurations preserve $1 / 16$ of the supersymmetry and hence lead to three-dimensional field theories with $\mathcal{N}=1$ supersymmetry, after suitably decoupling gravity. The 3-cycles will be taken to have constant curvature: we will consider three-spheres, hyperbolic 
three-space and possible quotients of these spaces by freely acting discrete subgroups of the corresponding isometry groups. Note that such quotients of hyperbolic space can be compact.

Following the strategy in [2, 3] we construct the ten and eleven dimensional solutions, by first constructing solutions in minimal $\mathrm{D}=7$ gauged supergravity. When the topological mass vanishes, corresponding to NS fivebranes, we find explicit solutions. The solutions are singular both in $D=7$ and in $D=10,11$. For the case of the three-sphere, the $S U(2)$-gauge fields in $\mathrm{D}=7$ have a meron form and moreover we do not find a supersymmetric instanton. This is surprising in the sense that one has the reverse situation in Yang-Mills theory where the instantons are supersymmetric and the merons are non-supersymmetric. At the end of the paper we comment on the possibility of the singularities of the solutions being resolved by a non-supersymmetric instanton and speculate on the connection with spontaneous supersymmetry breaking of pure $\mathcal{N}=1 \mathrm{SYM}$ in $\mathrm{D}=3$. When the topological mass is non-vanishing, corresponding to M-fivebranes, for the case of hyperbolic spaces we find a flow to an $A d S_{4}$ region. This implies that at low-energies the corresponding twisted field theory on the M-fivebrane flows to a superconformal field theory in $\mathrm{D}=3$, at least in the large $N$ limit.

The plan of the rest of this paper is as follows. We begin with some preliminary discussion of the twisted theories arising from the fivebranes wrapping associative three-cycles. The supergravity solutions are presented in section 3 and the paper closes with some discussion of our results.

\section{General Comments on the Twisted Fivebrane Theories}

Consider the Type IIB NS 5-brane wrapped on an associative 3-cycle $\Sigma$ in a manifold $M$ of $G_{2}$ holonomy. The world-volume of the fivebrane is then $\mathbb{R}^{1,2} \times \Sigma$. The nontrivial part of the spin connection on the worldvolume is a connection on the spin bundle $S$ of $\Sigma$. This is an $S U(2) \subset \operatorname{Spin}(1,5)$ bundle. The normal bundle to the fivebrane in the $G_{2}$-manifold is four dimensional and given by $N=S \otimes V$ where $S$ is the spinor bundle of $\Sigma$ and $V$ is a rank two $S U(2)$-bundle [14]. From this information the appropriate twisting can be deduced (see [15]): one identifies the structure group of $S, S U(2)_{\Sigma}$, with one of the $S U(2)$ factors, $S U(2)_{L}$ say, in the $\operatorname{Spin}(4) \cong S U(2)_{L} \times S U(2)_{R}$ R-symmetry group of the fivebrane.

The spin content of the twisted theory can thus be specified by giving the trans- 
formations under $\operatorname{Spin}(2,1) \times S U(2)_{D} \times S U(2)_{R}$, where $S U(2)_{D}$ is the diagonal of $S U(2)_{\Sigma} \times S U(2)_{L}$. Now recall that the fields of the flat fivebrane consist 4 scalars transforming as $(\mathbf{1}, \mathbf{2}, \mathbf{2})$, under $\operatorname{Spin}(5,1) \times S U(2)_{L} \times S U(2)_{R}$, fermions transforming as $(4,2,1)+(\overline{4}, \mathbf{1}, 2)$ and a six-dimensional vector field. By decomposing the $\operatorname{Spin}(5,1)$ representations into $\operatorname{Spin}(2,1) \times S U(2)_{\Sigma}$ representations we can then deduce the representations of the twisted theory. We find that the six-dimensional vector field gives rise to a three-dimensional vector field plus three scalars transforming as $(\mathbf{3}, \mathbf{1})$ of $S U(2)_{D} \times S U(2)_{R}$. The 4 scalars give rise to scalars transforming as $(\mathbf{2}, \mathbf{2})$ : they have become sections of the normal bundle mentioned above. Finally, the fermions transform as $(\mathbf{2}, \mathbf{3}, \mathbf{1})+(\mathbf{2}, \mathbf{1}, \mathbf{1})+(\mathbf{2}, \mathbf{2}, \mathbf{2})$ of $\operatorname{Spin}(2,1) \times S U(2)_{D} \times S U(2)_{R}$. The spinors that generate the supersymmetries on the NS fivebrane transform in exactly the same way and $(\mathbf{2}, \mathbf{1}, \mathbf{1})$ are the preserved supersymmetries corresponding to $\mathcal{N}=1$ in $\mathrm{D}=3$.

When $N$ IIB NS-fivebranes wrap an associative 3-cycle they give rise to this twisted theory with all fields in the adjoint of $U(N)$. At energies much less than the size of the cycle the theory will reduce to an $\mathcal{N}=1$ supersymmetric field theory in $\mathrm{D}=3$. The low-energy degrees of freedom correspond to $\mathcal{N}=1 \mathrm{SYM}$ in $\mathrm{D}=3$ but there could be extra massless fields arising from zero modes of the normal bundle: harmonic sections of $S \otimes V$. In this paper we are only considering associative 3-cycles that are 3-spheres, hyperbolic 3-space or quotients of these spaces. An example of a non-compact $G_{2}$-holonomy manifold with an associative $S^{3}$ was described in [25, 26]. The 7-manifold is in fact the total space of the spin bundle of $S^{3}, S\left(S^{3}\right)$. $S U(2)$ bundles on $S^{3}$ are trivial so $S\left(S^{3}\right)$ is homeomorphic to $\mathbb{R}^{4} \times S^{3}$. The associative $S^{3}$ is identified as the zero section of $S$. In this case, as pointed out in [14, it is obvious that the normal bundle to this sphere is just $S$ itself and hence $V$ must be trivial. One can immediately conclude that there are no zero modes since there are no harmonic spinors on the three-sphere. However, we do not know if this is the generic situation for associative three-spheres. Similarly one can consider $S\left(\mathbb{H}^{3}\right)$ which admits a $G_{2^{-}}$ holonomy metric defined on an open subset [25]. Again, $V$ is trivial, but in this case because $\mathbb{H}^{3}$ has negative constant curvature it is possible that harmonic spinors may exist.

When M-fivebranes wrap an associative cycle there is one dimension which is neither tangent to the fivebrane world-volume nor tangent to the manifold with $G_{2}$ holonomy. The $R$-symmetry is now $S O(5)$ but the twisting involves embedding the $S U(2)$ spin connection in an $S O(4)$ subgroup in the same way as for the IIB fivebrane. Consequently the analysis above allows us to conclude that this theory also preserves 
$\mathcal{N}=1$ supersymmetry in $\mathrm{D}=3$. For a single fivebrane we expect to get a single $\mathcal{N}=1$ $\mathrm{D}=3$ scalar superfield and possibly some extra massless states arising from the normal bundle. It is less clear what we will get when we have $N$ coincident fivebranes since we do not have an explicit six-dimensional Lagrangian for the fivebrane theory. If we wrap one of the uncompactified world-volume directions on a circle we would get the twisted theory in 2 spacetime dimensions arising from the $U(N)$ D4-brane theory wrapping the associative 3-cycle.

\section{Supergravity Solutions}

Following [2, 3] our strategy for constructing $\mathrm{D}=10$ and $\mathrm{D}=11$ supergravity solutions corresponding to string theory and M-fivebranes wrapped on associative 3-cycles of constant curvature is to first construct the solutions in $\mathcal{N}=1 \mathrm{D}=7$ gauged supergravity.

The bosonic field content of $\mathcal{N}=1 \mathrm{D}=7$ gauged supergravity [16] consists of a metric $g$, dilaton $\phi$, a three-form potential $A_{3}$ and $S U(2)$ gauge fields $A \equiv A^{a}\left(\tau^{a} / 2\right)$, where $\tau^{a}$ are Pauli-matrices. The fermions are made up of a dilatino $\lambda$ and a gravitino $\psi_{\mu}$, each an eight component complex $S U(2)$ Majorana spinor. The Lagrangian for the bosonic fields in the string-frame is given by

$$
\begin{aligned}
\mathcal{L}= & \sqrt{g} e^{-2 \phi}\left[\left(R-\frac{1}{8} F_{\mu \nu}^{a} F^{a \mu \nu}+4 \partial \phi^{2}\right)-\left(\frac{h^{2}}{2} e^{-4 \phi}-4 h e^{-2 \phi}-4\right)\right] \\
& -\frac{1}{2} e^{2 \phi} * G \wedge G+\frac{1}{4} F^{a} \wedge F^{a} \wedge A_{3}-\frac{h}{2} G \wedge A_{3}
\end{aligned}
$$

where $G=d A_{3}$ is the four-form field strength and $F=d A+i A \wedge A \equiv F^{a}\left(\tau^{a} / 2\right)$ is the $S U(2)$ field strength. The Einstein metric is related to the string metric via $g_{E}=e^{-4 \phi / 5} g$. The potential $V=e^{4 \phi / 5}\left[h^{2} / 2 e^{-4 \phi}-4 h e^{-2 \phi}-4\right]$ is drawn in Figure 1. Note that we have set the gauge coupling constant of [16] to $\sqrt{2}$ and we have rescaled the topological mass $h$ by a factor of 8 for convenience. When the topological mass vanishes we can dualise the 3 -form potential and rewrite the Lagrangian in terms of a 2 -form potential $B$ as

$$
\mathcal{L}=\sqrt{g} e^{-2 \phi}\left[R-\frac{1}{8} F_{\mu \nu}^{a} F^{a \mu \nu}+4 \partial \phi^{2}-\frac{1}{3} H_{\mu \nu \rho} H^{\mu \nu \rho}+4\right]
$$

with $d H=\frac{1}{8} F^{a} \wedge F^{a}$. Bosonic solutions to the equations of motion preserve supersymmetry if the supersymmetry variation of the dilatino and gravitino vanish:

$$
\begin{aligned}
\delta \lambda & =\left[\not \partial \phi-\frac{i}{4} \Gamma^{\mu \nu} F_{\mu \nu}+\frac{1}{48} e^{2 \phi} \Gamma^{\mu \nu \rho \sigma} G_{\mu \nu \rho \sigma}-h e^{-2 \phi}+1\right] \epsilon=0 \\
\delta \psi_{\mu} & =\left[D_{\mu}+i A_{\mu}-\frac{i}{2} F_{\mu \rho} \Gamma^{\rho}+\frac{1}{96} e^{2 \phi} \Gamma_{\mu}^{\nu \rho \sigma \delta} G_{\nu \rho \sigma \delta}-\frac{h}{4} e^{-2 \phi} \Gamma_{\mu}\right] \epsilon=0
\end{aligned}
$$


where the spinor $\epsilon$ carries an $S U(2)$ index upon which the Pauli matrices act.

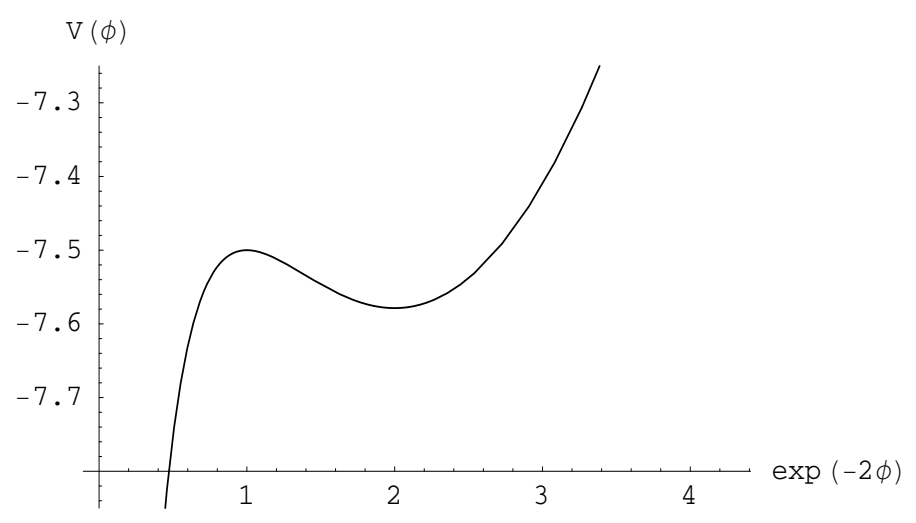

Figure 1: Scalar potential of $\mathrm{D}=7$ gauged supergravity with $h=1$.

To orient ourselves, we first recall some simple configurations that preserve supersymmetry. For vanishing topological mass, the linear dilaton solution

$$
\begin{aligned}
d s^{2} & =d s^{2}\left(\mathbb{E}^{1,5}\right)+d r^{2} \\
\phi & =-r
\end{aligned}
$$

with $F=G=0$ preserves $1 / 2$ of the supersymmetry. Uplifting to $\mathrm{D}=10 \mathrm{using}$ the formulae in [17, 18] we get

$$
\begin{aligned}
d s^{2} & =d s^{2}\left(\mathbb{E}^{1,5}\right)+d r^{2}+\frac{1}{4}\left(\omega_{1}^{2}+\omega_{2}^{2}+\omega_{3}^{2}\right) \\
\phi & =-r \\
H^{N S} & =\frac{1}{8} \omega_{1} \wedge \omega_{2} \wedge \omega_{3}
\end{aligned}
$$

where $\omega_{a}$ are left invariant one-forms on a 3 -sphere which we take to satisfy $d \omega_{1}=$ $\omega_{2} \wedge \omega_{3}$, and cyclic. This solution preserves $1 / 2$ of the $\mathcal{N}=1$ supersymmetry. It is also a supersymmetric solution of IIA/B supergravity preserving $1 / 2$ supersymmetry and corresponds to the near horizon limit of the IIA/B NS-fivebrane solution.

When the topological mass is non-vanishing it is more natural to use the Einsteinframe. The potential for the scalar-field $\phi$ has a supersymmetric maximum at $e^{-2 \phi}=$ $1 / h$ giving rise to the $A d S_{7}$ solution preserving all supersymmetry:

$$
\begin{aligned}
d s_{E}^{2} & =R^{2}\left[\frac{d u^{2}}{u^{2}}+u^{2} d s^{2}\left(\mathbb{E}^{1,5}\right)\right] \\
e^{-2 \phi} & =\frac{1}{h}
\end{aligned}
$$


with the AdS radius given by $R=2 / h^{1 / 5}$. Using the formulae in 19 this solution uplifts to $A d S_{7} \times S^{4}$ in $\mathrm{D}=11$ which is the near horizon limit of the M-fivebrane solution.

\subsection{Fivebranes wrapped on three-spheres}

To find more general solutions corresponding to IIB fivebranes and M-fivebranes wrapped on associative three-spheres, we consider an ansatz of the form

$$
\begin{aligned}
d s^{2} & =e^{2 f}\left[d \xi^{2}+d r^{2}\right]+a^{2} \sigma_{1}^{2}+b^{2} \sigma_{2}^{2}+c^{2} \sigma_{3}^{2} \\
A & =\alpha \sigma_{1}\left(\frac{\tau^{1}}{2}\right)+\beta \sigma_{2}\left(\frac{\tau^{2}}{2}\right)+\gamma \sigma_{3}\left(\frac{\tau^{3}}{2}\right) \\
A_{3} & =k \sigma_{1} \wedge \sigma_{2} \wedge \sigma_{3}-l d \xi^{0} \wedge d \xi^{1} \wedge d \xi^{2}
\end{aligned}
$$

with $f, \alpha, \beta, \gamma, k, l$ functions of $r$ only and $\sigma_{a}$ a basis of left invariant one-forms on $S^{3}$ satisfying $d \sigma_{1}=\sigma_{2} \wedge \sigma_{3}$ and cyclic permutations. Throughout the paper $d \xi^{2}$ refers to $d s^{2}\left(\mathbb{E}^{1,2}\right)$. When $a, b, c$ are not all equal, the fivebranes would be wrapping a squashed three-sphere. Note that in the special case that

$$
\frac{b^{2}+c^{2}-a^{2}}{2 b c}=\alpha, \quad \frac{a^{2}+c^{2}-b^{2}}{2 a c}=\beta, \quad \frac{a^{2}+b^{2}-c^{2}}{2 a b}=\gamma
$$

the $S U(2)$ gauge fields are equal to the components of the spin connection on the squashed 3-sphere directions. More precisely, in the frame given by $\left(e^{0}, \ldots, e^{6}\right)=$ $\left(e^{f} d \xi^{0}, e^{f} d \xi^{1}, e^{f} d \xi^{2}, e^{f} d r, a \sigma_{1}, b \sigma_{2}, c \sigma_{3}\right)$, we then have $\omega^{5}{ }_{6}=\left(\left(b^{2}+c^{2}-a^{2}\right) / 2 b c\right) \sigma_{1}=A^{1}$ and similarly $\omega^{6}{ }_{4}=A^{2}, \omega^{4}{ }_{5}=A^{3}$. This is the expected twisting for associative threespheres as discussed in the last section.

Upon substituting this ansatz (without assuming (3.8)) into (3.3) we have only found supersymmetric configurations with non-vanishing gauge-fieldst when the fourform is trivial, $k=l=0$, the squashed 3-spheres are round, $a=b=c$, and $\alpha=\beta=\gamma=1 / 2$. Note that these restrictions do indeed satisfy (3.8). Specifically,

$$
\begin{aligned}
d s^{2} & =e^{2 f}\left[d \xi^{2}+d r^{2}\right]+a^{2}\left(\sigma_{1}^{2}+\sigma_{2}^{2}+\sigma_{3}^{2}\right) \\
A & =\frac{1}{2}\left[\sigma_{1}\left(\frac{\tau^{1}}{2}\right)+\sigma_{2}\left(\frac{\tau^{2}}{2}\right)+\sigma_{3}\left(\frac{\tau^{3}}{2}\right)\right] \\
A_{3} & =0
\end{aligned}
$$

\footnotetext{
${ }^{1}$ When the topological mass vanishes there is a supersymmetric solution with vanishing gaugefields, $\alpha=\beta=\gamma=0$. It has $f=k=0, a=b=c=$ constant, $\phi=\left(1+1 / 4 a^{2}\right)^{1 / 2} r$ and $G=\left(e^{-2 \phi} / a\right) d \xi^{0} \wedge d \xi^{1} \wedge$ $d \xi^{2} \wedge d r$. When uplifted to $\mathrm{D}=10$ it gives rise to the $1 / 4$ supersymmetric IIA/B solution corresponding to the near horizon limit of two NS fivebranes intersecting on a string that was discussed in 20 .
} 
is a supersymmetric solution to the equations of motion provided that the functions $a, f, \phi$ solve the differential equations:

$$
\begin{aligned}
\frac{a^{\prime}}{a} e^{-f}-\frac{1}{4 a^{2}}-\frac{h}{2} e^{-2 \phi} & =0 \\
e^{-f} \phi^{\prime}-\frac{3}{16 a^{2}}-h e^{-2 \phi}+1 & =0 \\
2 e^{-f} f^{\prime}-h e^{-2 \phi} & =0
\end{aligned}
$$

These configurations preserve $1 / 8$ of the 16 supercharges. If $\gamma^{a}$ are gamma matrices with respect to the above mentioned frame, the preserved supersymmetries satisfy $\gamma^{3} \epsilon=i \gamma^{56} \tau^{1} \epsilon=i \gamma^{64} \tau^{2} \epsilon=i \gamma^{45} \tau^{3}=\epsilon$ (note that the last condition is implied by the second and third conditions.). The spinor $\epsilon$ has a radial dependence given by $\epsilon=e^{f / 2} \epsilon_{0}$ for constant $\epsilon_{0}$. Since the spinors are independent of the coordinates on the three-sphere, these solutions are also supersymmetric on $S^{3} / \Gamma$, where $\Gamma$ is a discrete subgroup of $S O(4)$, the isometry group of $S^{3}$, that acts freely and discontinuously.

It is interesting to note that the gauge-field is half pure-gauge or a meron [21] (for a recent discussion see 22]). Explicitly, by definition of the left-invariant one-forms $\sigma_{a}$, we have $A=-(i / 2) \sigma_{a}\left(i \tau^{a} / 2\right)=-(i / 2) U^{-1} d U$ where $U$ is an arbitrary element of $S U(2)$. In Yang-Mills theory merons are singular gauge-fields that are not BPS but solve the second-order equations of motion. Moreover the singularities at the origin and at infinity can be resolved by adding a half-instanton. Here we have a somewhat reverse situation in that the meron is part of a BPS configuration and we have not been able to find corresponding supersymmetric instanton configurations. As such it would seem that the singularities in the gauge field cannot be resolved by adding half-instantons while preserving supersymmetry.

\subsubsection{Vanishing topological mass}

Let us first consider the case of vanishing topological mass, $h=0$. In this case we can easily integrate (3.10) to obtain the explicit solution

$$
\begin{aligned}
d s^{2} & =d \xi^{2}+d r^{2}+\frac{r}{2}\left(\sigma_{1}^{2}+\sigma_{2}^{2}+\sigma_{3}^{2}\right) \\
A & =\frac{1}{2}\left[\sigma_{1}\left(\frac{\tau^{1}}{2}\right)+\sigma_{2}\left(\frac{\tau^{2}}{2}\right)+\sigma_{3}\left(\frac{\tau^{3}}{2}\right)\right] \\
e^{2 \phi} & =e^{-2 r} r^{3 / 4} e^{2 \phi_{0}} \\
A_{3} & =0
\end{aligned}
$$

This solution has a curvature singularity at the origin as one might expect from the singularities of the meron gauge-field. For example, the Ricci-scalar is given by 
$R=3 / r$. In addition to the 3 -dimensional Poincare invariance, the solution is also invariant under $\mathrm{SO}(4)$ symmetry. (The round 3-sphere is obviously invariant and the gauge fields are up to an $\mathrm{SU}(2)$ gauge transformation.)

This solution can be uplifted to a solution of $\mathcal{N}=1$ supergravity in $\mathrm{D}=10$ using the formulae in [17, 18]. Explicitly we get

$$
\begin{aligned}
d s^{2} & =d \xi^{2}+d r^{2}+\frac{r}{2}\left(\sigma_{1}^{2}+\sigma_{2}^{2}+\sigma_{3}^{2}\right)+\frac{1}{4}\left[\nu_{1}^{2}+\nu_{2}^{2}+\nu_{3}^{2}\right] \\
e^{2 \phi} & =e^{-2 r} r^{3 / 4} e^{2 \phi_{0}} \\
H^{N S} & =\frac{1}{32}\left[\sigma_{2} \wedge \sigma_{3} \wedge \nu_{1}+\sigma_{3} \wedge \sigma_{1} \wedge \nu_{2}+\sigma_{1} \wedge \sigma_{2} \wedge \nu_{3}\right]+\frac{1}{8} \nu_{1} \wedge \nu_{2} \wedge \nu_{3}
\end{aligned}
$$

with $\nu_{a} \equiv \omega_{a}-\sigma_{a} / 2$ and $\omega_{a}$ the left invariant one-forms on a 3 -sphere introduced before. We have directly checked that this solution admits Killing spinors of $\mathcal{N}=1$ supergravity in $\mathrm{D}=10$ :

$$
\begin{aligned}
\delta \lambda & =\left(\gamma^{M} \partial_{M} \phi-\frac{1}{6} H_{M N P} \gamma^{M N P}\right) \epsilon=0 \\
\delta \psi_{M} & =\left(D_{M}-\frac{1}{4} H_{M N P} \gamma^{N P}\right) \epsilon=0
\end{aligned}
$$

provided that $\epsilon$ is constant and satisfies

$$
\gamma^{3567} \epsilon=\gamma^{3648} \epsilon=\gamma^{3459} \epsilon=\epsilon
$$

in the frame $\left(e^{0}, \ldots, e^{9}\right)=\left(d \xi^{0}, d \xi^{1}, d \xi^{2}, d r,(r / 2)^{1 / 2}\left[\sigma_{1}, \sigma_{2}, \sigma_{3}\right],(1 / 2)\left[\nu_{1}, \nu_{2}, \nu_{3}\right]\right)$. These projections can be recast in the following elegant way (see, e.g., equations (11) and (78) of [23]):

$$
\frac{2}{3}\left(\gamma_{i j}+\frac{1}{4} \psi_{i j k l} \gamma^{k l}\right) \epsilon=0
$$

where $i, j, k, l \in\{3,4,5,6,7,8,9\}$ and the four-form $\psi$ is $G_{2}$-invariant with non-zero components given by:

$$
\begin{aligned}
+1 & =\psi_{4578}=\psi_{3459}=\psi_{4679}=\psi_{3567}=\psi_{5689} \\
-1 & =\psi_{3789}=\psi_{3468}
\end{aligned}
$$

Under the decomposition $\operatorname{Spin}(9,1) \rightarrow \operatorname{Spin}(2,1) \times \operatorname{Spin}(7)$ the spinors decompose as $\mathbf{1 6} \rightarrow(\mathbf{2}, \mathbf{8})$. We can further decompose $\operatorname{Spin}(7)$ under $G_{2}$ with $\mathbf{8} \rightarrow \mathbf{1}+\mathbf{7}$. Equation (3.15) asserts that $\epsilon$ is $G_{2}$-invariant.

This solution is also a solution of type IIA and type IIB supergravity, where it still preserves 2 supercharges, i.e. it now preserves $1 / 16$ of the supersymmetry. To 
see this recall that in the string-frame a bosonic IIA configuration with vanishing Ramond-Ramond fields is supersymmetric if (see, e.g., [24])

$$
\begin{aligned}
\delta \lambda_{\mp} & = \pm\left(\not \partial \phi \pm \frac{1}{6} H_{M N P} \gamma^{M N P}\right) \epsilon_{ \pm}=0 \\
\delta \psi_{ \pm} & =\left(D_{M} \pm \frac{1}{4} H_{M N P} \gamma^{N P}\right) \epsilon_{ \pm}=0
\end{aligned}
$$

We find that the solution breaks all $\epsilon_{+}$supersymmetries and preserves $1 / 8$ of the $\epsilon_{-}$ supersymmetries. This is exactly as expected: the $G_{2}$-holonomy metric will preserve spinors $\epsilon_{ \pm}$satisfying (3.14). If we wrap a IIA NS-fivebrane around the associative 3cycle in the directions $\{4,5,6\}$ we must impose $\gamma_{012456} \epsilon_{ \pm}=\epsilon_{ \pm}$which is only consistent with $\epsilon_{-}$. By explicit calculation, or simply by noting that we can obtain the IIB solution by performing a trivial T-duality in, the $\xi^{1}$ or $\xi^{2}$ direction, we conclude that as a solution of the IIB theory it also preserves $1 / 16$ of the supersymmetry. We can also trivially uplift the IIA solution to obtain a solution in $\mathrm{D}=11$ preserving $1 / 16$ of the supersymmetry.

The symmetries of the $\mathrm{D}=10$ solution consist of the $\mathrm{D}=2+1$ Poincare invariance as well as $S U(2)^{3}$ symmetry: the two left actions for which $\omega$ and $\sigma$ are left-invariant and an $S U(2)$ right-action which is the sum of the two-right actions. These isometries arise from the fact that the associative 3-cycle is a round three-sphere and that the normal bundle is not generic.

The asymptotic behaviour of the fivebrane is as one expects for a IIB fivebrane wrapping the three-sphere. Presumably the fact that the three-sphere is getting large is related to the fact that the $\mathrm{D}=2+1$ gauge coupling has dimension $1 / 2$. Note that just as in seven dimensions, the $\mathrm{D}=10$ solution is singular as $r \rightarrow 0$. For example, $H^{2}$ and the Ricci scalar diverge like $1 / r^{2}$. We will return to the issue of singularities in the last section.

Before closing this section, as somewhat of an aside, we report on a generalisation of the solution (3.12). Consider the ansatz

$$
\begin{aligned}
d s^{2} & =d \xi^{2}+d r^{2}+a^{2}\left(\sigma_{1}^{2}+\sigma_{2}^{2}+\sigma_{3}^{2}\right)+b^{2}\left[\nu_{1}^{2}+\nu_{2}^{2}+\nu_{3}^{2}\right] \\
H^{N S} & =l\left[\sigma_{2} \wedge \sigma_{3} \wedge \nu_{1}+\sigma_{3} \wedge \sigma_{1} \wedge \nu_{2}+\sigma_{1} \wedge \sigma_{2} \wedge \nu_{3}\right]+n \nu_{1} \wedge \nu_{2} \wedge \nu_{3}
\end{aligned}
$$

This preserves supersymmetry if $l=n / 4=C$ for constant $C$, provided that $a, b, \phi$ satisfy:

$$
\begin{aligned}
& \left(a^{2}\right)^{\prime}=\frac{b}{2}\left(1+\frac{8 C}{b^{2}}\right) \\
& \left(b^{2}\right)^{\prime}=b\left(1-\frac{b^{2}}{4 a^{2}}\right)\left(1-\frac{8 C}{b^{2}}\right)
\end{aligned}
$$




$$
\phi^{\prime}=\frac{3 C}{a^{2} b}-\frac{4 C}{b^{3}}
$$

For $C=1 / 32, b=1 / 2$ we recover our previous solution (3.12). For $C=0$, after introducing a new radial variable we find that the non-trivial seven metric is given

$$
d s_{7}^{2}=\left(1-\frac{k^{3}}{\rho^{3}}\right)^{-1} d \rho^{2}+\frac{\rho^{2}}{12}\left(\sigma_{1}^{2}+\sigma_{2}^{2}+\sigma_{3}^{2}\right)+\frac{\rho^{2}}{9}\left(1-\frac{k^{3}}{\rho^{3}}\right)\left[\nu_{1}^{2}+\nu_{2}^{2}+\nu_{3}^{2}\right]
$$

which is known to be a metric with $G_{2}$ holonomy [25, 26] and is the one discussed in section 2. We have not been able to find the exact solution to (3.19). However, we can establish the asymptotic behaviour. By analysing $d b^{2} / d a^{2}$ we see that for large $a^{2}$ we have $b^{2} \approx 4 a^{2} / 3$ and $\phi \approx$ constant. Using $a$ as a radial variable we then have asymptotically

$$
d s_{7}^{2}=12 d a^{2}+a^{2}\left(\sigma_{1}^{2}+\sigma_{2}^{2}+\sigma_{3}^{2}\right)+\frac{4 a^{2}}{3}\left[\nu_{1}^{2}+\nu_{2}^{2}+\nu_{3}^{2}\right]
$$

which is the large $\rho$ limit of (3.20). For small $a^{2}$ we have $b^{2} \approx 1 / a$ and $\phi \approx$ constant $+12 \mathrm{Ca}$ giving rise to the asymptotic metric

$$
d s_{7}^{2}=16 a^{3} d a^{2}+a^{2}\left(\sigma_{1}^{2}+\sigma_{2}^{2}+\sigma_{3}^{2}\right)+\frac{1}{a}\left[\nu_{1}^{2}+\nu_{2}^{2}+\nu_{3}^{2}\right]
$$

Note that these solutions are not solutions of minimal $\mathrm{D}=7$ gauged supergravity because they have another scalar field active.

\subsubsection{Non-vanishing topological mass}

To find supergravity solutions describing M-theory fivebranes wrapped on associative three-spheres we need to solve $(\underline{3.10})$ with $h \neq 0$. We have not been able to find exact solutions, but it is not too difficult to establish the asymptotic behaviour of the solutions. Dividing the second equation by the first and defining

$$
F=x^{2} e^{-2 \phi}, \quad x=a^{2}
$$

we can obtain the following differential equation

$$
\frac{d F}{d x}=\frac{5 F+16 x F}{4 x+8 h F}
$$

The behaviour of the orbits in the $(F, x)$-plane is illustrated in figure 2 . It is also useful to note that (3.10) implies

$$
\frac{d f}{d x}=\frac{h F}{x^{2}+2 h F x}
$$




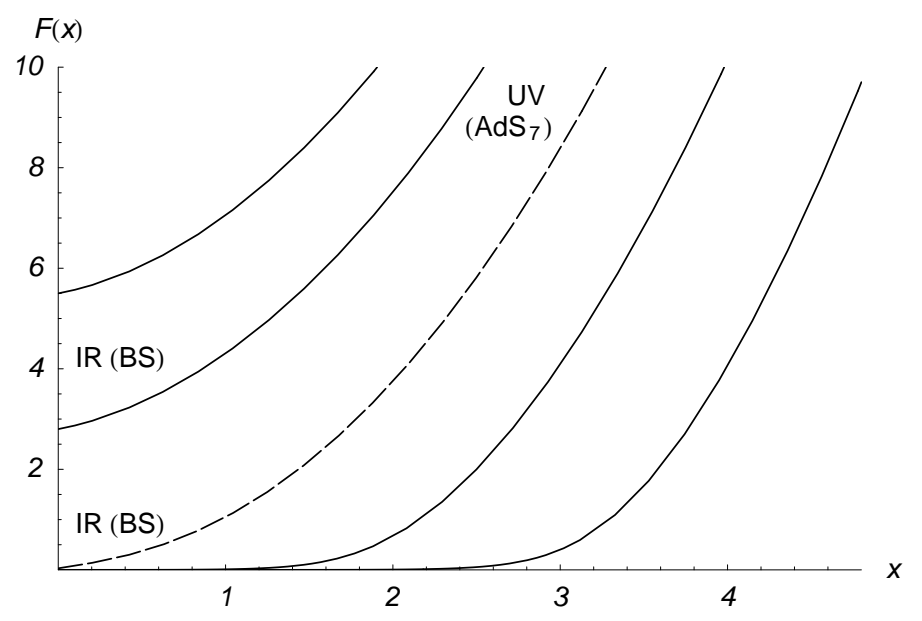

Figure 2: Behaviour of the orbits for the three-sphere when $h=1$. The $A d S_{7}$-type region is at $F \approx x^{2}$, for large $x$. The broken line is the separatrix. The singularity in the IR region is always of the bad type (BS).

For large $x$ we have $F \approx x^{2} / h-3 x / 8 h$. Switching the radial variable from $r$ to $a$ we then deduce that the asymptotic form of the Einstein metric and scalar are given by

$$
\begin{aligned}
d s_{E}^{2} & =\left(\frac{2}{h^{1 / 5}}\right)^{2}\left(\frac{d a^{2}}{a^{2}}+a^{2}\left[d \xi^{2}+\frac{1}{4}\left(\sigma_{1}^{2}+\sigma_{2}^{2}+\sigma_{3}^{2}\right)\right]\right) \\
e^{-2 \phi} & =\frac{1}{h}-\frac{3}{8 h a^{2}}
\end{aligned}
$$

(we have rescaled the coordinates $\xi$ ). This is the asymptotic behaviour that one expects for an M-fivebrane to be wrapped on a three-sphere: the dilaton is at the supersymmetric maximum of the potential, and the metric has the form of $A d S_{7}$ except that the slices of constant $a$ have $\mathbb{E}^{5,1}$ replaced with $\mathbb{E}^{2,1} \times S^{3}$. Moreover, the next to leading order behaviour of the dilaton is given by $\phi \approx(\ln h) / 2+3 /\left(16 a^{2}\right)$. This corresponds to the insertion of the boundary operator $\mathcal{O}_{\phi}$ of conformal dimension $\Delta=4$, since the falloff is like $1 / a^{6-\Delta}$. This operator is dual to $\Phi^{2}$ where $\Phi$ are the scalars in the tensor multiplet of the M-fivebrane theory (see e.g. [27]). The next order of the expansion corresponds to the expectation value of this operator 28].

For small $x$, the behaviour of $F$ depends on the value of $F(0)$. If it is non-vanishing we have $F \approx F_{0}+5 x / 8 h$. The dilaton and the Einstein metric are then asymptotically

$$
d s_{E}^{2}=4 F_{0}^{2 / 5}\left[\frac{a^{\frac{22}{5}}}{h^{2} F_{0}^{2}} d a^{2}+a^{\frac{2}{5}}\left(d \xi^{2}+\frac{1}{4}\left(\sigma_{1}^{2}+\sigma_{2}^{2}+\sigma_{3}^{2}\right)\right)\right]
$$




$$
e^{-2 \phi}=\frac{F_{0}}{a^{4}}
$$

If $F(0)$ vanishes we generically have $F \approx F_{0} x^{5 / 4}$ and

$$
\begin{aligned}
d s_{E}^{2} & =F_{0}^{2 / 5}\left[16 a^{\frac{7}{5}} d a^{2}+a^{-\frac{3}{5}} d \xi^{2}+a^{\frac{7}{5}}\left(\sigma_{1}^{2}+\sigma_{2}^{2}+\sigma_{3}^{2}\right)\right] \\
e^{-2 \phi} & =\frac{F_{0}}{a^{3 / 2}}
\end{aligned}
$$

The behaviour of the separatrix is $F \approx x / 8 h$, giving

$$
\begin{aligned}
d s_{E}^{2} & =\frac{1}{(8 h)^{2 / 5}}\left[\left(\frac{16}{5}\right)^{2} a^{\frac{6}{5}} d a^{2}+a^{-\frac{2}{5}} d \xi^{2}+a^{\frac{6}{5}}\left(\sigma_{1}^{2}+\sigma_{2}^{2}+\sigma_{3}^{2}\right)\right] \\
e^{-2 \phi} & =\frac{1}{8 h a^{2}}
\end{aligned}
$$

All of these metrics are singular. Note that (3.27) has $g_{00}$ decreasing as one approaches the singularity, while the others have it diverging. Before we conclude that the former is thus an example of a "good" singularity by the criteria of [2] (see also [29]), we should recall that the definition applied to the $\mathrm{D}=11$ solution. Using the formulae in [19], we see that the $\mathrm{D}=11$ metric will have the form (e.g., for $h=1$ )

$$
d s^{2}=\Delta^{1 / 3} d s_{E}^{2}+X^{3} \Delta^{1 / 3} d \theta^{2}+\frac{1}{4} \Delta^{-2 / 3} X^{-1} \cos ^{2} \theta\left(\nu_{1}^{2}+\nu_{2}^{2}+\nu_{3}^{2}\right)
$$

where $X=e^{2 \phi / 5}$

$$
\Delta=X^{-4} \sin ^{2} \theta+X \cos ^{2} \theta
$$

In all cases the 00 component of the eleven dimensional metric is divergent and hence the singularities are "bad" by the criteria of [2].

Finally, we note that for all solutions $e^{-2 \phi}$ starts from the supersymmetric maximum at $1 / h$, decreases in value before turning and then running off to infinity.

\subsection{Fivebranes wrapped on hyperbolic space}

Let us now more briefly describe what happens when we replace the three-sphere with possible quotients of hyperbolic three-space, $\uplus^{3} / \Gamma$. Here $\Gamma$ is a discrete subgroup of $S O(3,1) \cong P S L(2, \mathbb{C})$, the isometry group of $\uplus^{3} / \Gamma$, that acts freely and discontinuously. This includes cases when $\uplus^{3} / \Gamma$ is compact.

Consider the metric ansatz:

$$
d s^{2}=e^{2 f}\left[d \xi^{2}+d r^{2}\right]+4 \frac{a^{2}}{z^{2}}\left(d z^{2}+d x^{2}+d y^{2}\right)
$$


where $(z, x, y)$ are local coordinates on $\uplus^{3}$. We set the four-form $G$ to zero and take the $S U(2)$ gauge fields $A^{a}$ to be specified in terms of the spin connection via

$$
\begin{aligned}
& A^{1}=\omega^{5}{ }_{6}=0 \\
& A^{2}=\omega^{6}{ }_{4}=-\frac{1}{2 a} e^{6} \\
& A^{3}=\omega^{4}{ }_{5}=\frac{1}{2 a} e^{5}
\end{aligned}
$$

using the frame $\left(e^{f}\left(d \xi^{0}, d \xi^{1}, d \xi^{2}\right),(2 a / z)(z, x, y)\right)$. These configurations preserve $1 / 8$ of the supersymmetry if

$$
\begin{aligned}
\frac{a^{\prime}}{a} e^{-f}-\frac{1}{4 a^{2}}+\frac{h}{2} e^{-2 \phi} & =0 \\
e^{-f} \phi^{\prime}-\frac{3}{16 a^{2}}+h e^{-2 \phi}-1 & =0 \\
2 e^{-f} f^{\prime}+h e^{-2 \phi} & =0
\end{aligned}
$$

The spinors satisfy $i \gamma^{65} \tau^{1} \epsilon=i \gamma^{46} \tau^{2} \epsilon=i \gamma^{54} \tau^{3}=\gamma^{3} \epsilon=-\epsilon$ and their radial dependence is again given by $\epsilon=e^{f / 2} \epsilon_{0}$ for constant $\epsilon_{0}$. Since the spinors are independent of the coordinates on $\mathbb{H}^{3}$, these solutions are also supersymmetric on $\uplus^{3} / \Gamma$.

For vanishing topological mass, $h=0$, these equations can be integrated to give metric and dilaton:

$$
\begin{aligned}
d s^{2} & =d \xi^{2}+d r^{2}+\frac{2 r}{z^{2}}\left(d z^{2}+d x^{2}+d y^{2}\right) \\
e^{2 \phi} & =e^{2 r} r^{-3 / 4} e^{2 \phi_{0}}
\end{aligned}
$$

This solution can then be easily uplifted to $D=10$. Apart from the change in sign of the dilaton this is very similar to the case of the three-sphere. The solution is singular both in $\mathrm{D}=7$ and in $\mathrm{D}=10$.

For non-vanishing topological mass things are quite different from the case of the three-sphere. We first note that the differential equations (3.34) admit the exact solution $a^{2}=5 / 16, e^{-2 \phi}=8 / 5 h$ and $e^{f}=5 / 4 r$. In Einstein-frame we have

$$
d s^{2}=\left(\frac{8}{5 h}\right)^{\frac{2}{5}}\left[\frac{25}{16 r^{2}}\left(d \xi^{2}+d r^{2}\right)+\frac{5}{4 z^{2}}\left(d z^{2}+d x^{2}+d y^{2}\right)\right]
$$

corresponding to $A d S_{4} \times \mathbb{H}^{3} / \Gamma$. This can easily be lifted to $\mathrm{D}=11$ using (3.30), with $\nu_{a}=\omega_{a}-A_{a}$, and the formula for the four-form in [19].

We can get further insight into the solutions of (3.34) by again analysing the differential equation for $F=x^{2} e^{-2 \phi}, x=a^{2}$. We now have

$$
\frac{d F}{d x}=\frac{-5 F+16 x F}{-4 x+8 h F}
$$


Notice that this is the same equation as (3.10) after $x \rightarrow-x$. The behaviour of the orbits for the region of interest here is illustrated in figure 3 .

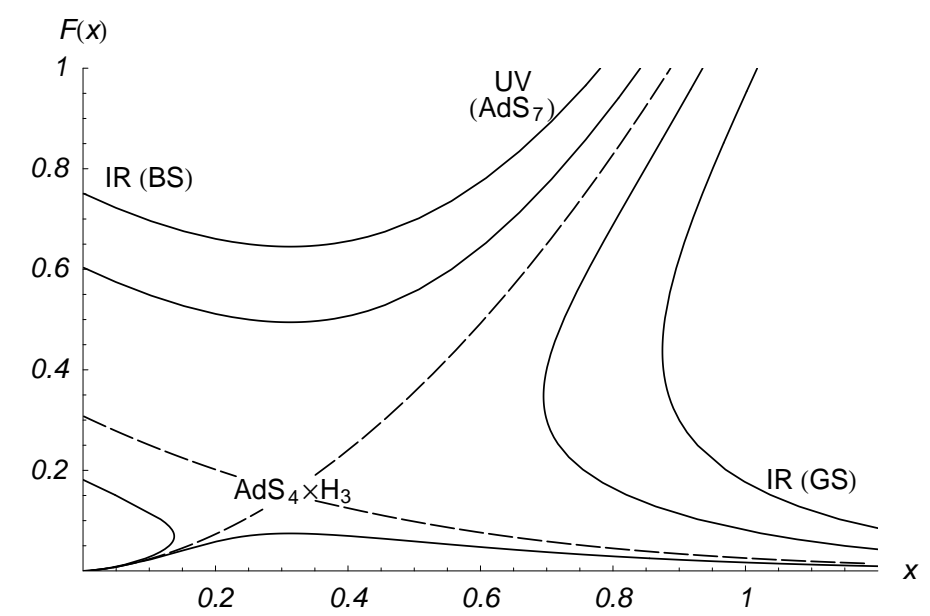

Figure 3: Behaviour of the orbits for hyperbolic spaces when $h=1$. The $A d S_{7}$-type region is at $F \approx x^{2}$, for large $x$, and flows to the IR fixed point or the good (GS) and bad (BS) singularities in the IR. The broken lines are the separatrices.

For large $x$ there are solutions that behave like $F \approx x^{2} / h+3 x / 8 h$ which give rise to the asymptotic solution

$$
\begin{aligned}
d s_{E}^{2} & =\left(\frac{2}{h^{1 / 5}}\right)^{2}\left(\frac{d a^{2}}{a^{2}}+a^{2}\left[d \xi^{2}+\frac{1}{z^{2}}\left(d z^{2}+d x^{2}+d y^{2}\right)\right]\right) \\
e^{-2 \phi} & =\frac{1}{h}+\frac{3}{8 h a^{2}}
\end{aligned}
$$

as one expects for an M-fivebrane wrapping the hyperbolic space. The next to leading order behaviour of the dilaton is now $\phi \approx(\ln h) / 2-3 /\left(16 a^{2}\right)$, again corresponding to the insertion of the boundary operator $\mathcal{O}_{\phi}$ of dimension $\Delta=4$.

There are three different types of behaviour of the these solutions as $x$ decreases. Firstly, there is an orbit that ends up at the solution (3.36). When $\mathbb{H}^{3} / \Gamma$ is compact, this orbit thus corresponds to a flow "across dimensions" from the $A d S_{7}$-type region (3.38) to an $A d S_{4} \times \mathbb{H}^{3} / \Gamma$ region. This implies that the twisted field theory living on a M-fivebrane wrapped on compact $\uplus^{3} / \Gamma$ flows in the far IR to a new superconformal theory (at least for large $N$ ) whose dual is described by (3.38).

There is also a class of orbits in which for small $x, F$ asymptotes to a constant, $F_{0}$. These solutions give rise to the asymptotic metric for small $x$ of the form

$$
d s_{E}^{2}=4 F_{0}^{2 / 5}\left[\frac{a^{\frac{22}{5}}}{h^{2} F_{0}^{2}} d a^{2}+a^{\frac{2}{5}}\left(d \xi^{2}+\frac{1}{z^{2}}\left(d z^{2}+d x^{2}+d y^{2}\right)\right]\right.
$$




$$
e^{-2 \phi}=\frac{F_{0}}{a^{4}}
$$

These have a similar structure to (3.27). In particular, although $g_{00}$ is decreasing as we approach the singularity at $a=0$, the 00 component of the uplifted $\mathrm{D}=11$ metric is divergent and hence these are "bad" by the criteria of [2]. For these flows $e^{-2 \phi}$ monotonically increases from $1 / h$ to infinity.

Finally there is another class of orbits in which $F$ decreases as a function of $x$ then turns back on itself and decreases to zero for large $x$. At the end of these orbits the large $x$ behaviour for $F$ is of the form $F \approx F_{0} e^{-4 x}$. Although $a$ is not a good radial coordinate along the whole of these trajectories, it is good enough to describe the asymptotic behaviour and we find that they have "good" singularities by the criteria of [2]. For these flows $e^{-2 \phi}$ begins increasing from $1 / h$ before turning and running back to zero.

\section{Discussion}

We have found supergravity solutions that describe fivebranes wrapping associative 3-cycles that are either $S^{3}, \uplus^{3}$ or quotients of these spaces. For the M-fivebrane case we determined the general asymptotic behaviour of the solutions to the BPS equations. In the case of the M-fivebrane wrapping a hyperbolic three-space we have shown that there is a flow from an $A d S_{7}$ type region to an $A d S_{4} \times \mathbb{H}^{3} / \Gamma$ solution. For compact $\sharp^{3} / \Gamma$ this implies that at low-energies the wrapped M-fivebrane theory flows to a superconformal theory in $\mathrm{D}=3$ at least for large $N$. It would be interesting to study this theory in more detail. We also found a class of orbits with "good" singularities which presumably correspond to switching on a vev for the operator $\mathcal{O}_{\phi}$. For all other orbits, both for the three-sphere and for hyperbolic spaces, the singularities in the IR are "bad" by the criteria of [8]. It will be interesting to see if and how they can be resolved.

For the IIB NS-fivebrane theory we obtained exact solutions to the BPS equations and they are all singular in the IR. Let us speculate on how the singularities might be resolved for the case of the three-sphere. In section 2 we noted that after taking a suitable decoupling limit one expects that the IIB NS-fivebrane wrapped on an associative three-sphere should give rise to $\mathcal{N}=1$ supersymmetric Yang-Mills theory in the IR, at least for certain $G_{2}$ manifolds when the associative three-sphere is rigid. Witten has shown [30] that the Witten index vanishes for $\mathcal{N}=1 \mathrm{SYM}$ in $\mathrm{D}=3$

with vanishing Chern-Simons coupling and has provided circumstantial evidence that 
supersymmetry is actually spontaneously broken. If this is indeed the case, it is natural to suggest that our supergravity solution (3.12) describes this case and that the singularity can only be removed in a non-supersymmetric fashion. Recalling that the singularity is related to the meron gauge fields in $D=7$ gauged supergravity, it is plausible that the singularity can be removed by a supersymmetry breaking instanton. It would be interesting to construct such supergravity solutions and thereby, hopefully, demonstrate spontaneous breaking of supersymmetry in pure $\mathcal{N}=1 \mathrm{SYM}$ in $\mathrm{D}=3$.

We should comment that Witten has also shown [30] that for suitable ChernSimons couplings the Witten Index in $\mathcal{N}=1 \mathrm{SYM}$ is non-vanishing and hence supersymmetry is preserved. One might hope to be able to find supersymmetric gravity solutions describing this situation. Since the IIB NS fivebrane includes a coupling of the form $F \wedge F \wedge B_{N S} \sim \omega_{C S}(A) \wedge H$, where $F$ is the field strength of the gauge fields $A$ living on the fivebrane, one expects that the presence of NS three-form flux $H$ on the three-sphere would give rise to these theories. However, our original ansatz did allow for this type of possibility, but we did not find such a supersymmetric solution. We have not proven that our solutions are the only ones within our ansatz that preserve supersymmetry but we expect that a more general ansatz is probably needed to find these solutions, if they indeed exist.

In our approach, following [2, 3], we did not start with a manifold with $G_{2}$ holonomy and then construct a solution describing a fivebrane wrapping an associative 3 -cycle. Rather we built the solution all at once. This then raises the question about which $G_{2}$-holonomy manifolds we are considering in our final solutions. This does not seem to be a straightforward question to answer as it is not clear how to "switch off" the fivebrane flux. Nevertheless, it appears that the manifolds are $S\left(S^{3} / \Gamma\right)$ or $S\left(\mathbb{H}^{3} / \Gamma\right)$ of 25, 26] that we discussed in section 2. The evidence for this is as follows. Firstly, the decoupling limit that we take leads to a non-compact manifold: only the local description of the associative three-cycle is important. Secondly, the structure of the normal bundle of the associative cycles in $S\left(S^{3} / \Gamma\right)$ or $S\left(\mathbb{H}^{3} / \Gamma\right)$ correspond to our solutions. The generic normal bundle of an associative three-cycle has structure group $S O(4) \approx S U(2)_{L} \times S U(2)_{R}$ and the twisting requires an identification of the $S U(2)$ spin-connection on the cycle with one of the factors, $S U(2)_{L}$, say. Our solutions are constructed in minimal gauged supergravity which only has $S U(2)_{L}$ gauge fields and hence we can only construct solutions corresponding to associative threecycles with non-generic normal bundles. The normal bundles to the associative three cycles in $S\left(S^{3} / \Gamma\right)$ or $S\left(\mathbb{H}^{3} / \Gamma\right)$ also have the $S U(2)_{R}$ bundle trivial. Finally in section 3.1.1 we derived some generalised BPS equations that contain these manifolds as well 
as our solutions as special cases, for the case of vanishing topological mass. If we compare (3.20) and (3.12) it is interesting to note that associative 3-sphere in $S\left(S^{3}\right)$ gets shrunk when we add the fivebrane while the other asymptotic three-sphere that shrunk to zero size at the zero section gets blown up to finite size. The latter is necessary in order for there to be non-zero flux transverse to the wrapped brane. It is also interesting to note that while the $G_{2}$ invariant metric on $S\left(\mathbb{H}^{3} / \Gamma\right)$ is not complete, when we add the M-fivebrane flux we can get the regular solution $A d S_{4} \times \mathbb{H}^{3} / \Gamma$ uplifted to $\mathrm{D}=11$.

\section{Note Added:}

After this work was completed we became aware of 31] where they also found the $A d S_{4} \times \mathbb{H}^{3}$ solution of minimal $\mathrm{D}=7$ gauged supergravity. In addition this paper presented an $A d S_{4} \times \mathbb{H}^{3}$ solution for maximal $\mathrm{D}=7$ gauged supergravity. This solution is related to M-fivebranes wrapping special Lagrangian 3-cycles in Calabi-Yau threefolds as will be shown elsewhere 32 .

\section{Acknowledgements}

We thank Fay Dowker, Nikita Nekrasov, Paul Townsend, Daniel Waldram and especially Juan Maldacena for helpful discussions. JPG thanks the EPSRC for partial support. JPG and NK are supported in part by PPARC through SPG \#613.

\section{References}

[1] M. Bershadsky, C. Vafa and V. Sadov, D-Branes and Topological Field Theories, Nucl. Phys. B463 (1996) 420, hep-th/9511222.

[2] J. Maldacena and C. Nunez, Supergravity description of field theories on curved manifolds and a no go theorem, hep-th/0007018.

[3] J. M. Maldacena and C. Nunez, Towards the large $n$ limit of pure $N=1$ super Yang Mills, hep-th/0008001.

[4] M. Alishahiha and Y. Oz, AdS/CFT and BPS Strings in Four Dimensions, Phys.Lett. B465 (1999) 136, hep-th/9907206

[5] M. Cvetic, H. Lu, C.N. Pope, J.F. Vazquez-Poritz, AdS in Warped Spacetimes, Phys.Rev. D62 (2000) 122003, hep-th/0005246. 
[6] A. Fayyazuddin and D. J. Smith, Localized intersections of M5-branes and four-dimensional superconformal fiel d theories, JHEP 9904 (1999) 030, hepth/9902210.

[7] A. Fayyazuddin and D. J. Smith, Warped AdS near-horizon geometry of completely localized intersections of M5-branes, JHEP 0010 (2000) 023, hepth/0006060.

[8] B. Brinne, A. Fayyazuddin, S. Mukhopadhyay, D. J. Smith, Supergravity M5branes wrapped on Riemann surfaces and their QFT duals, hep-th/0009047.

[9] I. R. Klebanov and A. A. Tseytlin, Gravity duals of supersymmetric $S U(N) x$ $S U(N+M)$ gauge theories, Nucl. Phys. B578 (2000) 123, hep-th/0002159.

[10] I. R. Klebanov and M. J. Strassler, Supergravity and a confining gauge theory: Duality cascades and $\chi S B$-resolution of naked singularities, JHEP 0008 (2000) 052, hep-th/0007191.

[11] M. Cvetic, H. Lu and C. N. Pope, Brane resolution through transgression, hepth/0011023.

[12] J. Maldacena, The large N limit of superconformal field theories and supergravity, Adv. Theor. Math. Phys. 2 (1998) 231, hep-th/9711200.

[13] N. Itzhaki, J. M. Maldacena, J. Sonnenschein and S. Yankielowicz, Supergravity and the large $N$ limit of theories with sixteen supercharges, Phys. Rev. D58 (1998) 046004, hep-th/9802042.

[14] R. McLean, Deformations of Calibrated Sub-Manifolds, Comm. Anal. Geom. 6 (1998) 705-747, available at http://www.math.duke.edu/preprints/1996.htm].

[15] M. Blau and G. Thompson, Aspects of $N(T) \geq 2$ topological gauge theories and D-branes, Nucl. Phys. B492 (1997) 545, hep-th/9612143.

[16] P. K. Townsend and P. van Nieuwenhuizen, Gauged Seven-Dimensional Supergravity, Phys. Lett. B125 (1983) 41; L. Mezincescu, P. K. Townsend and P. van Nieuwenhuizen, Stability Of Gauged D $=7$ Supergravity And The Definition Of Masslessness In (Ads) In Seven-Dimensions, Phys. Lett. B143 (1984) 384.

[17] A. H. Chamseddine and W. A. Sabra, $D=7$ SU(2) gauged supergravity from $D$ $=10$ supergravity, Phys. Lett. B476 (2000) 415 hep-th/9911180. 
[18] M. Cvetic, H. Lu and C. N. Pope, Consistent Kaluza-Klein sphere reductions, Phys. Rev. D62 (2000) 064028 hep-th/0003286.

[19] H. Lu and C. N. Pope, Exact embedding of $N=1, D=7$ gauged supergravity in $D=11$, Phys. Lett. B467 (1999) 67 hep-th/9906168.

[20] P. M. Cowdall and P. K. Townsend, Gauged supergravity vacua from intersecting branes, Phys. Lett. B429 (1998) 281, hep-th/9801165.

[21] V. de Alfaro, S. Fubini and G. Furlan, A New Classical Solution Of The YangMills Field Equations, Phys. Lett. B65 (1976) 163; C. G. Callan, R. Dashen and D. J. Gross, A Mechanism For Quark Confinement, Phys. Lett. B66 (1977) 375; C. G. Callan, R. Dashen and D. J. Gross, Toward a theory of the strong interactions, Phys. Rev. D17 (1978) 2717.

[22] N. Drukker, D. J. Gross and N. Itzhaki, Sphalerons, merons and unstable branes in AdS, Phys. Rev. D62 (2000) 086007 hep-th/0004131.

[23] J. P. Gauntlett, N. D. Lambert and P. C. West, Branes and calibrated geometries, Commun. Math. Phys. 202 (1999) 571 hep-th/9803216.

[24] A. Dabholkar, J. P. Gauntlett, J. A. Harvey and D. Waldram, Strings as Solitons \& Black Holes as Strings, Nucl. Phys. B474 (1996) 85 hep-th/9511053.

[25] R.L. Bryant and S. Salamon, On the construction of some complete metrics with exceptional holonomy, Duke Math. J. 58 (1989) 829.

[26] G. W. Gibbons, D. N. Page and C. N. Pope, Einstein Metrics On $S^{3}, R^{3}$ And $R^{4}$ Bundles, Commun. Math. Phys. 127 (1990) 529.

[27] V. L. Campos, G. Ferretti, H. Larsson, D. Martelli and B. E. Nilsson, A study of holographic renormalization group flows in $d=6$ and $d=3$, JHEP 0006 (2000) 023, hep-th/0003151.

[28] V. Balasubramanian, P. Kraus and A. Lawrence, Bulk vs. boundary dynamics in anti-de Sitter spacetime, Phys. Rev. D 59 (1999) 046003, hep-th/9805171.

[29] S. S. Gubser, Curvature singularities: The good, the bad, and the naked, hepth/0002160.

[30] E. Witten, Supersymmetric index of three-dimensional gauge theory, hepth/9903005. 
[31] M. Pernici and E. Sezgin, Spontaneous Compactification Of Seven-Dimensional Supergravity Theories, Class. Quant. Grav. 2 (1985) 673.

[32] J.P. Gauntlett, N. Kim and D.J. Waldram, M-Fivebranes Wrapped on Supersymmetric Cycles, hep-th/0012195. 